\title{
Research on Inhibition Effect of Vortex-Induced Vibration of Submarine Pipeline with Horizontal Spoilers
}

\author{
Liu Long*, Dong Dongshan and Mei Xiao
}

Logistics Engineering College, Shanghai Maritime University, Shanghai, 201306, China

\begin{abstract}
This study discusses hydrodynamic effects of horizontal spoilers fixed on submarine pipelines. Numerical calculations are conducted to investigate the influences of the spoiler on flow profile, vortex shedding frequency, drag coefficients and lift coefficients. The calculation results demonstrate that attached horizontal spoilers could change the wake flow and enhance the self-burial of the pipeline. Moreover, the vortex-inhibition effects of different sizes of spoiler are discussed. The length of horizontal spoilers between 1.0D 1.5D has the optimal effect. The results would be promising for the application to submarine pipelines.
\end{abstract}

Keywords: Hydrodynamic effects, pipeline, spoiler, submarine, vortex shedding.

\section{INTRODUCTION}

Nowadays, the exploitation of deep-sea oil and gas resources are paid great attention. Submarine pipelines are very important for the production system of ocean oil and gas, as the pipelines can transport safely and quickly. Because of the complex ocean environment, the vortex-induced vibration may cause pipelines breakdown. To protect offshore pipelines from possible damage caused by excessive hydrodynamic forces or human activities, pipelines are often buried to a certain depth below the seabed.

The process of self-burial of offshore pipelines may occur naturally, and past research has found the attached spoiler on the top of a pipeline is one of the effective means to enhance this process and realize the vortex inhabitation effect [1,2]. Hulsbergen and Bijker [3] indicate that selfburial process for a pipeline with a top-attached spoiler is about 10 times faster than that without it. Kwon [4] proposes a drag reduction device that uses three ribbons attached 120 degrees apart to vertical pipe, and discuss the effect of cylinder's vortex shedding and drag resistance. Yang and Zhang [5] discuss the effect of suppressing efficiency of helical stakes, which is mainly affected by the stake geometry and coverage length.

On the research of marine pipeline's suppression devices, the sensibility to flow direction need to be mainly considered. Therefore different types of spoilers are invented to realize the pipeline's self-burial process and suppress the vortex induced vibration. Chiew [6] draws up a conclusion that the submarine pipeline with the spoilers increased the rate and extent of the seabed scour. Wang and Zhao [7] conduct numerical simulation to testify the different influences of spoilers on pipelines self-burial behavior. The installation of vertical spoilers has proved that it can realize the submarine pipeline's self-burial and had a good effect on the

\footnotetext{
*Address correspondence to this author at the Logistics Engineering College, Shanghai Maritime University, Shanghai, 201306, P.R. China; Tel: 86-02138282668; E-mail: longliu @ shmtu.edu.cn
}

suppression of the vortex induced vibration, but studies on the horizontal spoilers are less.

This paper proposes the horizontal spoiler to inhabit the submarine pipeline's vortex induced vibration. Using the Fluent software, the change of laminar flow are discussed under the condition of different gap between the pipeline and seabed. Moreover, the paper also discusses the effect of different spoilers' lengths on the vortex-induced vibration of pipeline and proposes a more reasonable size of horizontal spoilers.

Nowadays, the exploitation of deep-sea oil and gas resources are paid great attention. Submarine pipelines are very important for the production system of ocean oil and gas, as the pipelines can transport safely and quickly. Because of the complex ocean environment, the vortex-induced vibration may cause the pipelines' breakdown. To protect offshore pipelines from possible damage caused by excessive hydrodynamic forces or human activities, pipelines are often buried to a certain depth below the seabed.

\section{NUMERICAL MODEL}

The basic equations that are used in this study are the two-dimensional Navier-Stokes equations for unsteady incompressible viscous flows. The Navier-Stokes equations are solved using the pressure Poisson equation method [8].

\subsection{Calculation Domain}

Fig. (1) shows the flow field of the submarine pipeline with a horizontal spoiler. In this flow filed, the pipeline's diameter is set to $D$. The pipe locates at a distance of 10 diameters from the inlet boundary $(L D=8 D)$. As previous numerical tests of an isolated pipeline at different Reynolds numbers have revealed that the lateral boundaries have no significant effect on the global parameters if they are located at least eight diameters away from the pipe center [9]. The distance of 10-times diameters is set for the top lateral 
boundary $(T D=10 D)$. The outlet boundary locates at a distance of 15 diameters $(R D=15 D)$ from the pipe center

Moreover, the length of the spoiler is set to $S$; the location of bottom wall boundary relative to the pipe changes for different gap ratios $(G)$.

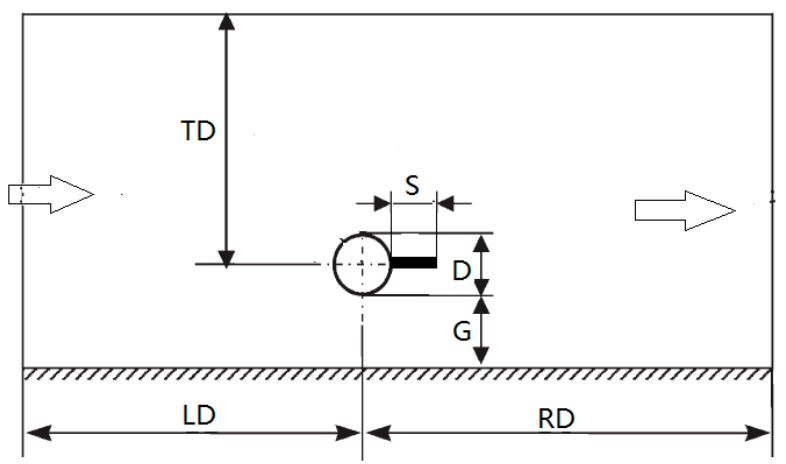

Fig. (1). Layout of the flow domain.

\subsection{Boundary and Initial Conditions}

The seawater's temperature is set to $15^{\circ} \mathrm{C}$. Different velocity and pressure boundary conditions are applied to different boundaries.

At the inlet, the transverse velocity component is set to zero and the longitudinal velocity is set to uniform as 0.0025 $\mathrm{m} / \mathrm{s}$ based on laminar boundary layer theory. The symmetry boundary condition is prescribed on the top lateral boundary. The pipe surface is on the non-slip wall boundary condition and zero velocity components are specified.

For the inlet and wall boundaries, the pressure is determined according to the momentum equations normal to the boundaries.

\subsection{Meshing Grid}

The grid for this numerical study is generated using an algebraic method using ICEM software. An H-type grid was constructed in the center section of the flow domain where the pipe is located. Fig. (2) is the meshing grids of the flow field.

The grid near the pipe and the wall boundary is changed according to different gap ratios. The size of grid is selected based on the mesh-dependence study of a uniform flow at different Reynolds numbers. The minimum radial spacing between the first circumferential grid line and the pipe surface must not be greater than $0.004 D$.

All the calculations are carried out with the time-step 0.001 using ANSYS CFX software. Tests have been done to demonstrate this time-step is small enough to ensure the solutions' precision is relatively independent on time-steps.

\section{NUMERICAL COMPUTATION}

Numerical tests have been carried out for flow around a pipe with and without a horizontal spoiler as the flow velocity is set to $0.0025 \mathrm{~m} / \mathrm{s}$. The gap ratio $G / D$ between pipe and the smooth is set in the range of $0 \sim 1.0$. For the case of pipe with a spoiler, the lengths of spoiler are taken as $1.5 D$.
Due to the limitation of the computer facility, relatively low Reynolds numbers are investigated in present research.

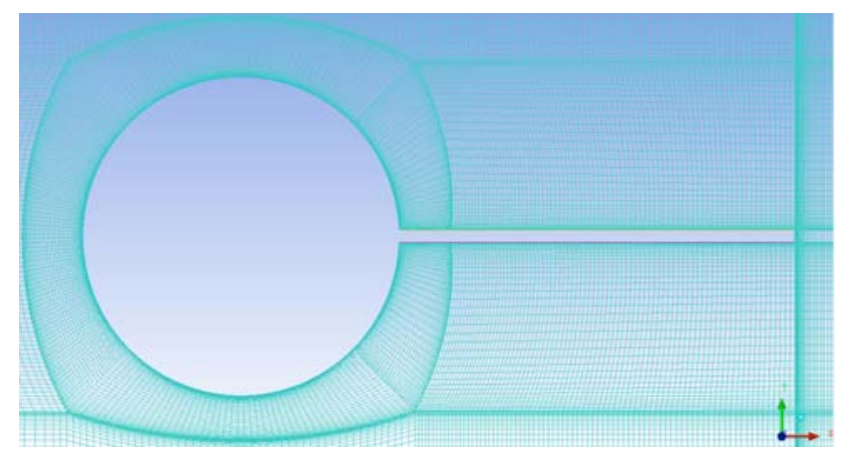

Fig. (2). Grid mesh near the pipeline and spoiler.

\subsection{Effect of Horizontal Spoiler on Pipeline's Flow}

Compared with the streamline in the wake flow of pipeline without the horizontal spoiler under different gaps, as shown in Fig. (3), the middle-lines of the vortex shedding with horizontal spoiler, which is shown in Fig. (4), are increased. After the split-flow, the upper and the lower side fluid confluence are delayed because of the barrier of spoiler.

So the vortex does not drop near the pipeline. but goes back to the downstream region, which the pipeline is not surrounded by the strong vortex. It can be concluded that horizontal spoiler has significant effects on the flow field distribution around the pipes.

\subsection{Effect of Horizontal Spoiler on Drag Coefficients}

As shown in previous section, the addition of a horizontal spoiler on the pipe near a smooth wall has a significant effect on flow field, and this influence would affect forces acting on the pipe subsequently. In this section, we will discuss horizontal spoiler's effects on drag coefficients.

The drag coefficient $C_{d}$ is defined as:

$$
C_{d}=\frac{F_{d}}{0.5 \rho U^{2} A}
$$

In this equation, $F_{d}$ is the drag force; $A$ is the area of the pipeline wall vertical to the flow direction; $\rho$ is the density of the seawater; and $U$ the flow velocity.

Fig. (5) shows the curves of drag coefficients changing with horizontal spoilers as the gap is $0.5 \mathrm{D}$.

Except the irregular fluctuation of drag coefficient as the gap is $0.1 D$, the changes of drag coefficient become stable quickly, and are less than 2. (Table 1) lists numerical results of average drag coefficients changing with and without horizontal spoilers under different gaps.

The results illustrate that the addition of a horizontal spoiler decreases the drag on the pipe significantly nearly for all gap ratios. This decrease creates a little wake, which is due to the delayed separation of flow on the right side of the spoiler. 

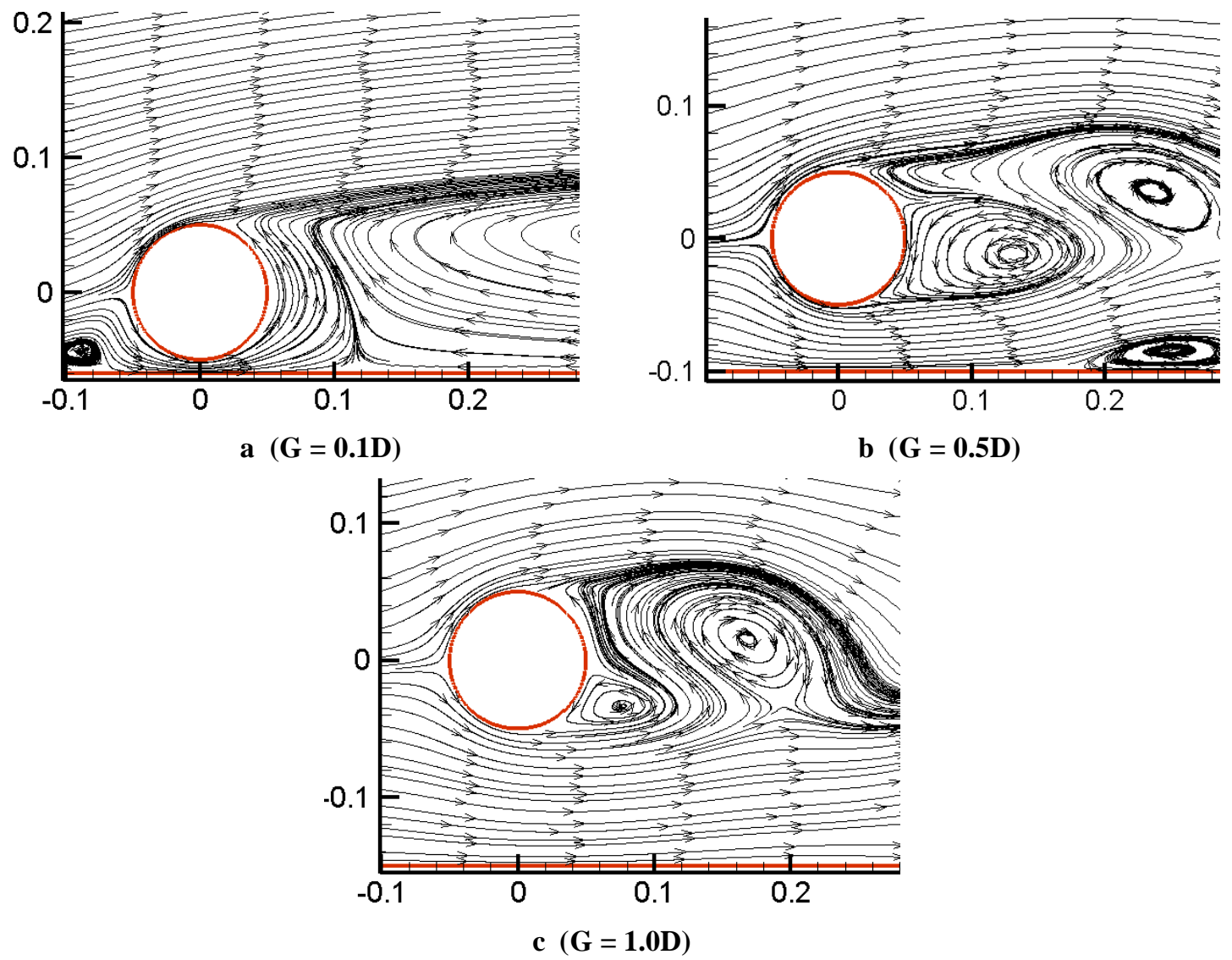

Fig. (3). Pipeline's streamlines without the spoilers under different gaps.

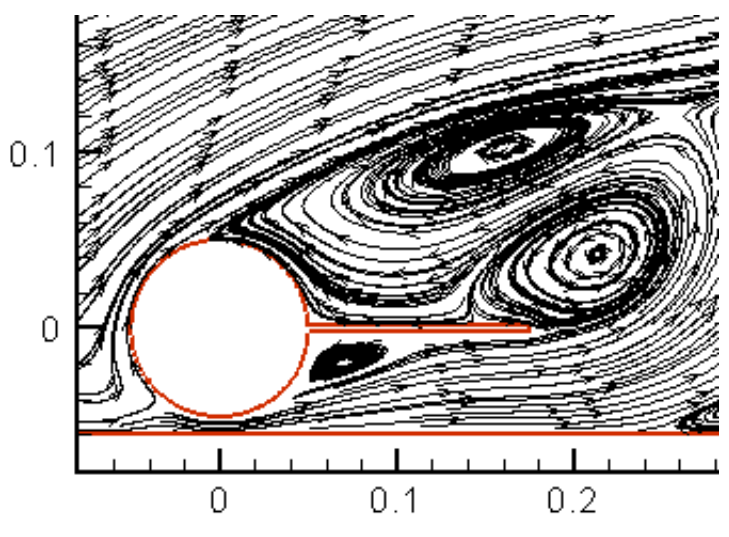

a $(\mathrm{G}=0.1 \mathrm{D})$
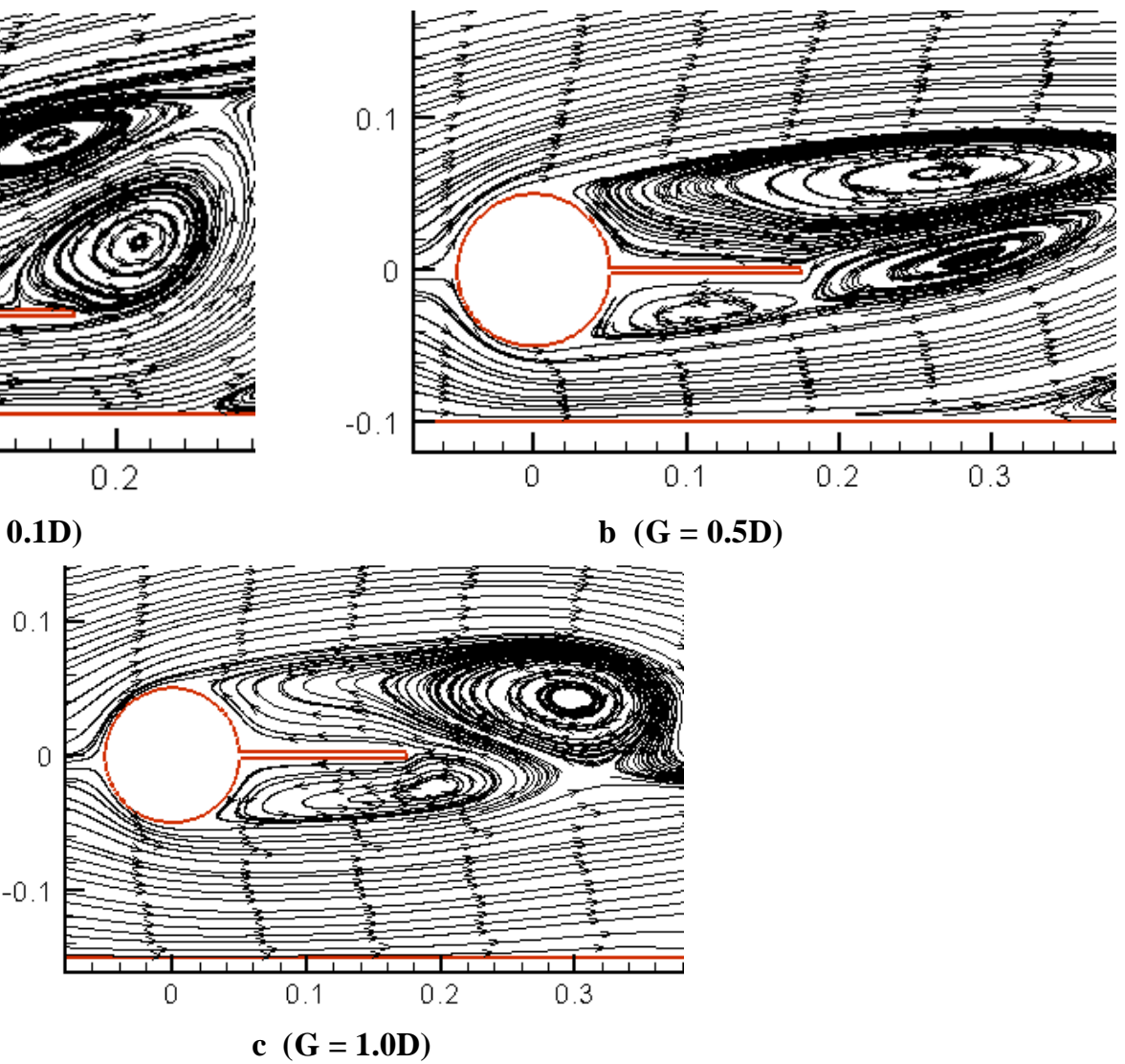

Fig. (4). Pipeline's streamlines with spoilers under different gaps. 


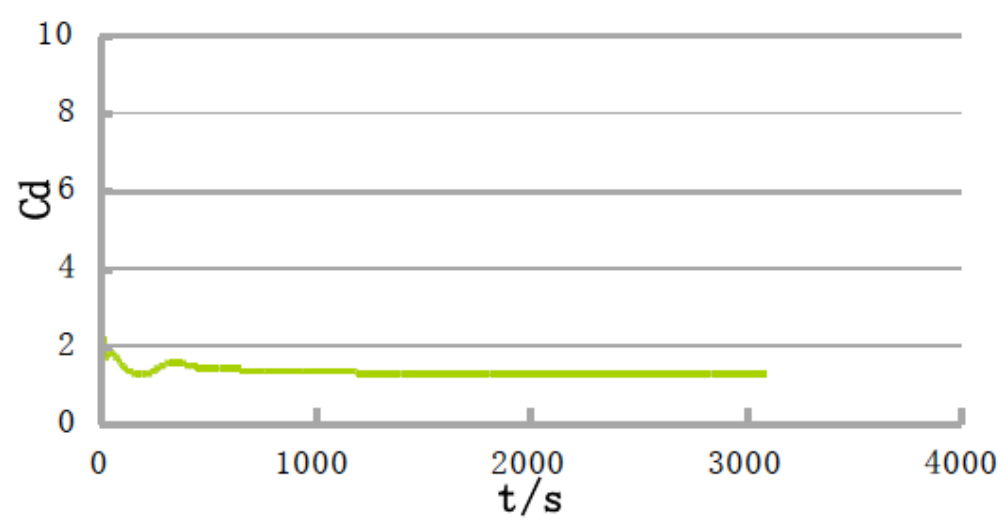

Fig. (5). The curve of drag coefficient with spoilers.

Table 1. Drag coefficients under differnt Gap Ratios.

\begin{tabular}{|c|c|c|}
\hline Gap ratio & $\begin{array}{c}\text { With horizontal } \\
\text { spoilers }\end{array}$ & Without spoilers \\
\hline \hline 0.1 & 1.2586 & 1.2491 \\
\hline 0.3 & 1.3987 & 1.4480 \\
\hline 0.5 & 1.4045 & 1.5251 \\
\hline 1.0 & 1.3178 & 1.5373 \\
\hline
\end{tabular}

\subsection{Effect of Horizontal Spoiler on Lift Coefficients}

The lift coefficient $C_{l}$ is defines as:

$$
C_{l}=\frac{F_{l}}{0.5 \rho A U^{2}}
$$

where $F_{l}$ is the lift force.

Fig. (6) and Fig. (7) show the curves of lift coefficients without and with horizontal spoilers as the gap is $0.5 \mathrm{D}$, respectively.

When the horizontal spoilers are not installed as shown in Fig. (6), the amplitudes of the lift coefficient changing with time are obviously large, and the average values of the lift coefficient are positive, which can easily cause the pipeline to float and buckle failure.

Because of the installation of horizontal spoilers, it has made a great interference impact on the vortex shedding of pipeline wake flow and obviously decreased the vertical force on the pipeline. As is shown in Fig. (7), the lift curves are more regular under the other gap conditions.

\subsection{Effect of Horizontal Spoiler on Vortex Shedding Frequencies}

The vortex shedding frequency is defined as:

$$
f_{s}=\frac{S_{t} U}{D}
$$

where $S_{t}$ is a dimensionless number, which is the function of Reynolds number and structural cross-section shape parameter.
Fig. (8) and Fig. (9) are the curves of power spectrum density of vortex shedding without and with horizontal spoilers as the gap is $0.5 \mathrm{D}$, respectively.

For the pipeline without spoilers as shown in Fig. (8), when the gap ratios are increasing, the vortex shedding frequencies corresponding to the peak value of energy are increasing.

For the pipeline with horizontal spoilers, the frequency changes of the vortex shedding in the wake flow are obvious. Moreover, the vortex shedding frequencies are short and its changes are not evident with the change of gap.

\subsection{Optimal Design of Horizontal Spoilers}

This section would discuss the different lengths of horizontal spoilers on the suppression effect of pipeline's vortex induced vibration when the gap ratios are 0.5 and $1.0 \mathrm{D}$, respectively.

In order to ensure the reliability of the mode's comparative analysis, the flow calculation domain and other parameters remain unchanged. The spoilers' length is changes from 0 to $1.5 \mathrm{D}$.

(Tables 2,3 ) are calculation results of drag coefficient, lift coefficient and the frequency of vortex shedding with different lengths of horizontal spoilers, respectively.

We can see that except that $S=0.25 \mathrm{D}$, the fluctuation amplitudes of lift coefficients are less than that of pipeline without horizontal spoilers. The average value and maximum of the lift coefficients of the pipeline with spoilers is less than that of pipeline without spoilers. Moreover, with the increasing of the spoiler's length, the maximum of the lift coefficient decrease constantly.

For two gap ratios, different lengths of spoilers make the obvious difference on the effect of drag coefficients reduction, which are over $8 \%$.

For vortex shedding frequencies, with the increasing of the spoiler's length, the frequencies decrease from 0.0055 to 0.0016 , which illustrates that vortex shedding frequencies depend on the spoiler's length. The increase of spoiler's length is beneficial to that the frequency of vortex shedding and could inhibit the vortex induced vibration.

Moreover, the length of horizontal spoiler between 1.0D 1.5D could obtain the optimal inhibition effect. 


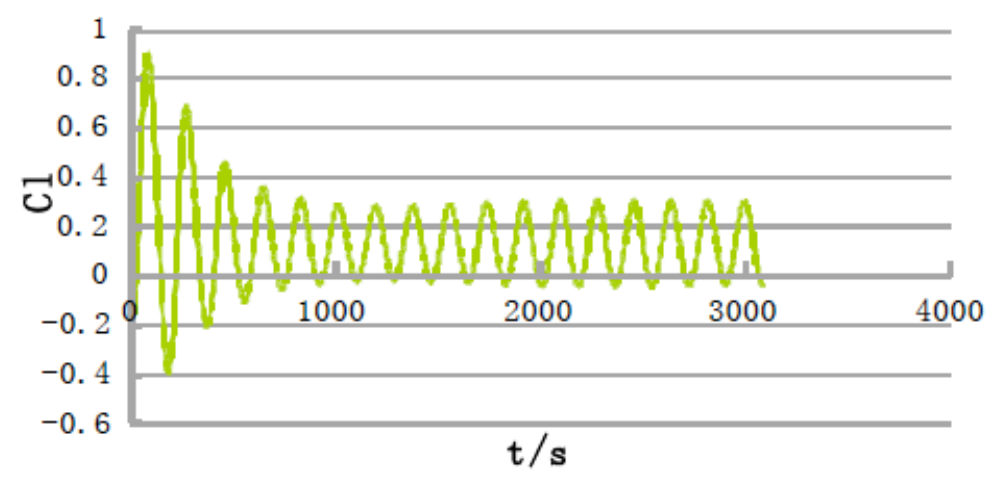

Fig. (6). Lift coefficients without spoilers.

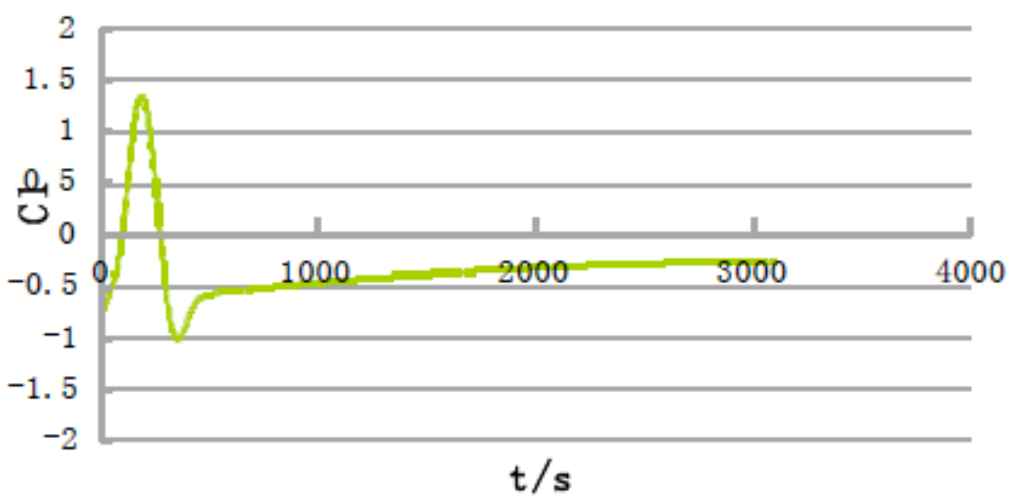

Fig. (7). Lift coefficients with horizontal spoilers.

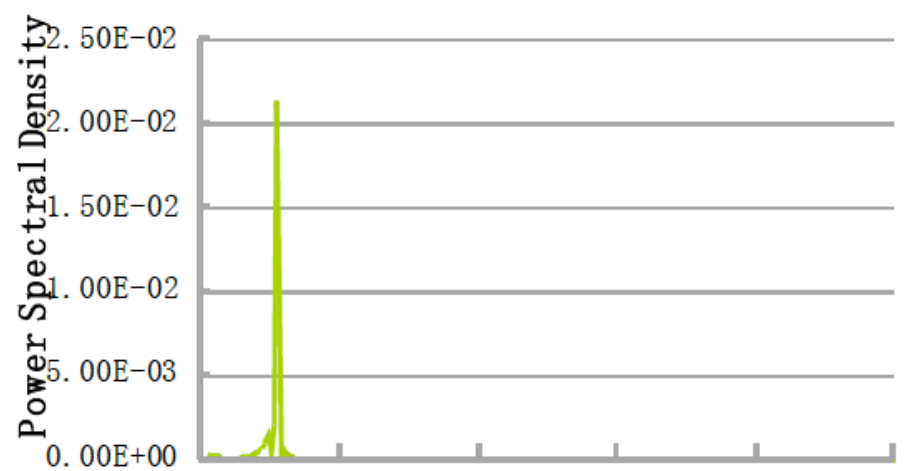

0. $00 \mathrm{E}+00$ 1. $.00 \mathrm{E}-022.00 \mathrm{E}-023.00 \mathrm{E}-024.00 \mathrm{E}-02$ 5. 00E-02 Frequency/Hz

Fig. (8). Power spectrum of vortex shedding under different gap without spoilers.

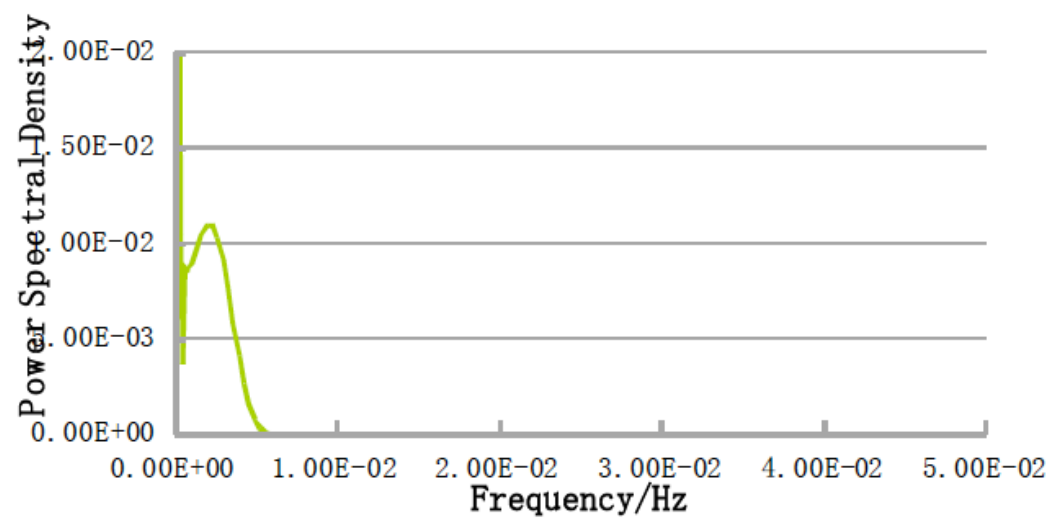

Fig. (9). Power spectrum of vortex shedding under different gap with spoilers. 
Table 2. Comparison of drag coefficient, lift coefficient and the frequency of vortex shedding $(G=0.5 D)$.

\begin{tabular}{|c|c|c|c|c|c|c|}
\hline G/D & Cd average value & $\begin{array}{c}\text { The effect of drag } \\
\text { reduction }\end{array}$ & $\begin{array}{c}\text { Cl fluctuation } \\
\text { amplitude }\end{array}$ & Cl (Maximun) & $\begin{array}{c}\text { The effect of lift } \\
\text { reduction }\end{array}$ & $\begin{array}{c}\text { Frequency of } \\
\text { vortex shedding }\end{array}$ \\
\hline \hline 0 & 1.5678 & - & 0.3469 & 0.3072 & - \\
\hline 0.25 & 1.3894 & $11.38 \%$ & 0.4319 & 0.2983 & $-24.5 \%$ & 0.0055 \\
\hline 0.50 & 1.3499 & $13.90 \%$ & 0.0328 & 0.0587 & $90.54 \%$ & 0.0052 \\
\hline 0.75 & 1.3521 & $13.76 \%$ & 0.0048 & -0.0161 & $98.62 \%$ & 0.0033 \\
\hline 1.00 & 1.3641 & $12.99 \%$ & 0.0059 & -0.1074 & -0.2547 & $85.13 \%$ \\
\hline 1.25 & 1.3897 & $11.36 \%$ & 0.0516 & -0.5142 & $98.79 \%$ & 0.0020 \\
\hline 1.50 & 1.4045 & $1042 \%$ & 0.0042 & 0.0016 \\
\hline
\end{tabular}

Table 3. Comparison of drag coefficient, lift coefficient and the frequency of vortex shedding (G=1.0D).

\begin{tabular}{|c|c|c|c|c|c|c|}
\hline G/D & Cd average value & $\begin{array}{c}\text { The effect of drag } \\
\text { reduction }\end{array}$ & $\begin{array}{c}\text { Cl fluctuation } \\
\text { amplitude }\end{array}$ & Cl (Maximun) & $\begin{array}{c}\text { The effect of lift } \\
\text { reduction }\end{array}$ & $\begin{array}{c}\text { Frequency of } \\
\text { vortex shedding }\end{array}$ \\
\hline \hline 0 & 1.5556 & - & 0.9499 & 0.5987 & 0.0055 \\
\hline 0.25 & 1.4272 & $8.25 \%$ & 0.8314 & 0.5226 & $12.71 \%$ & $32.74 \%$ \\
\hline 0.50 & 1.3759 & $11.55 \%$ & 0.6361 & 0.4027 & $53.87 \%$ & 0.0052 \\
\hline 0.75 & 1.3391 & $13.92 \%$ & 0.4624 & 0.2762 & 0.1804 & 0.0049 \\
\hline 1.00 & 1.3262 & $14.75 \%$ & 0.3902 & 0.0868 & $85.50 \%$ & 0.0029 \\
\hline 1.25 & 1.3213 & $15.06 \%$ & 0.3828 & -0.0201 & 0.0026 \\
\hline 1.50 & 1.3178 & $15.29 \%$ & 0.4293 & 0.0023 \\
\hline
\end{tabular}

\section{CONCLUSION}

In this paper, horizontal spoiler of submarine pipelines is proposed to inhibit vortex-induced vibration. Firstly, the calculation model of pipeline's flow field with horizontal spoiler is established. Then, compared with the pipeline without horizontal spoiler, we discuss the horizontal spoiler effect on the pipeline's flow wake. Moreover, the inhibition effects of different sizes of spoiler on vortex-induced vibration are discussed.

When the horizontal spoiler is fixed, the upper and the lower side fluid confluence is delayed, and the vortex does not drop near the pipeline but goes back to the downstream region, which the pipeline is not surrounded by the strong vortex. So the pipeline is not easily damaged by vortex induction vibration. Meanwhile, drag coefficients and lift coefficients get less. So the vortex induced vibration of pipeline can be suppressed evidently.

By numerical computation of different lengths of horizontal spoilers, we can obtain that he length of horizontal spoiler between 1.0D 1.5D has the optimal effect.

The horizontal spoilers are promising for the application to offshore structures. Further work is required to understand the effects of the relative sizes of horizontal spoilers and cylindrical pipe. However, extensions of the experimental test are essential for applications to real structures.

\section{CONFLICT OF INTEREST}

The authors confirm that this article content has no conflict of interest.

\section{ACKNOWLEDGEMENTS}

This work was supported by Innovation Program of Shanghai Municipal Education Commission (12YZ118).

\section{REFERENCES}

[1] T. Sarpkaya, "A critical review of the intrinsic nature of vortexinduced vibrations", Journal of Fluids and Structures, vol. 19, pp. 389-448, Oct. 2004

[2] B. M. Sumer, C. Truelsen, T. Sichmann, "On set of scour below pipelines and self-burial", Coastal Engineering, vol. 42, pp. 313315, Oct. 2001.

[3] C. H. Hulsbergen, R. Bijker, "Effect of spoilers on submarine pipeline stability", Offshore Technology Conference, vol. 6154, pp. 337-350, Mar. 1989.

[4] S.H. Kwon, J. W. Cho, J.S. Park , "The effects of drag reduction by ribbons attached to cylindrical pipes", Ocean Engineering, vol. 29, pp. 1945-1958, Sep. 2002.

[5] J. D. Yang, X.L. Zhang, "Helical stakes-vortex induced vibration suppression devices of deepwater risers", Ocean Technology, vol. 29, no. 4, pp. 88-91, Apr. 2010. 
[6] Y. M. Chiew, "Effect of spoilers on wave-induced scour at submarine pipelines", Port Coastal, and Ocean Engineering, vol. 119, no. 4, pp. 417-428, Apr. 1993.

[7] X. C Wang, J.P. Zhao, "Effect of spoiler on self-burial behaviour of submarine pipeline by numerical simulation analysis", Conical Piled Monopod, vol. 35, no. 6, pp. 8-10, Jun. 2007.
[8] C. Lei, L. Cheng, S. Armfield, K. Kavanagh, "Vortex shedding suppression for flow over a circular cylinder near a plane boundary", Ocean Engineering, vol. 27, no. 10, pp.1109-1127, Oct. 2000.

[9] B. Chen, L.Cheng, "Numerical investigation of three-dimensional flow around a free-spanned pipeline", Proceeding of the twelfth International Offshore and Polar Engineering Conference, San Francisco, 2002, pp. 61-67.

Received: September 22, 2014

Revised: December 01, 2014

Accepted: December 15, 2014

(C) Long et al.; Licensee Bentham Open.

This is an open access article licensed under the terms of the Creative Commons Attribution Non-Commercial License (http://creativecommons.org/licenses/by-nc/3.0/) which permits unrestricted, non-commercial use, distribution and reproduction in any medium, provided the work is properly cited. 